BULL. AUSTRAL. MATH. SOC.

VOL. 22 (1980), 365-372.

\title{
OSCILLATING PROPERTIES OF THE SOLUTIONS \\ OF A CLASS OF NEUTRAL TYPE FUNCTIONAL DIFFERENTIAL EQUATIONS
}

\author{
A. I. Zahariev and D.D. Bainov
}

\begin{abstract}
The present paper deals with some oscillating and asymptotic properties of the functional differential equations of the form $x^{\prime \prime}(t)+\lambda x^{\prime \prime}(t-\tau)+F\left(t, x(t-\tau), x^{\prime}(t-\tau)\right)=0$

where $\lambda$ is an arbitrary positive constant and $\tau>0$ is a constant delay.
\end{abstract}

The present paper deals with some oscillating and asymptotic properties of some functional differential equations of the form

$$
x^{\prime \prime}(t)+\lambda x^{\prime \prime}(t-\tau)+F\left(t, x(t-\tau), x^{\prime}(t-\tau)\right)=0
$$

where $\lambda$ is an arbitrary positive constant, and $\tau>0$ is a constant delay. We should point out that the oscillating properties of second order functional differential equations when $\lambda=0$ have been studied in many papers. Shevelo's monograph [2] contains a detailed bibliography on that subject.

We introduce some definitions.

DEFINITION 1. We shall consider as a solution of equation (1) every function

Received 29 April 1980. 


$$
x(t)= \begin{cases}x^{0}(t), & t \in\left[t_{0}-\tau, t_{0}\right], \\ \tilde{x}(t), & t \in\left[t_{0},+\infty\right),\end{cases}
$$

for every $t \geq t_{0}, t_{0} \in \mathbb{R}^{1}$, where $x^{0}(t) \in c^{\perp}\left(\left[t_{0}-\tau, t_{0}\right], \mathbb{R}^{1}\right)$, $\tilde{x}(t) \in C^{2}\left(\left[t_{0},+\infty\right), \mathbb{R}^{1}\right)$ and by $x^{\prime}\left(t_{0}\right)$ we shall denote a right derivative.

By $W$ we shall denote the set of solutions of equation ( 1 ), satisfying the condition $x(t) \neq 0$ in every interval $[\bar{t},+\infty), \bar{t} \geq t_{0}$, and we shall assume that $W \neq \emptyset$.

DEFINITION 2. The solution $x(t) \in W$ will be called oscillating if it changes its sign in every interval $[\bar{t},+\infty), \bar{t} \geq t_{0}$.

THEOREM 1. Let the conditions (A) be satisfied:

Al. the function $F(t, u, v): \mathbb{D} \rightarrow \mathbb{R}^{1} \quad\left(\mathbb{D}=\left[t_{0},+\infty\right) \times \mathbb{R}^{2}\right)$ is continuous, $F(t, 0,0) \equiv .0$ for $t \geq t_{0}$ and it satisfies the inequality

$$
F(t, u, v) \operatorname{sign} u \geq p(t) f(u)
$$

for alz points $(t, u, v) \in \mathbb{D}$;

A2. the function $f(u): \mathbb{R}^{1} \rightarrow \mathbb{R}^{1}$ is continuous, $u f(u)>0$ for $u \neq 0$ and $\inf |f(u)|>0$ for $|u| \geq \varepsilon>0$;

A3. the function $p(t):\left[t_{0},+\infty\right) \rightarrow[0,+\infty)$ is continuous and for every closed set $E$ whose intersection with every segment of the form $[t, t+2 \tau] \quad\left(t_{0} \leq t<+\infty\right)$ has a measure not smazler that $\tau$, the equality

$$
\int_{E} p(t) d t=+\infty
$$

holds.

Then every solution $x(t) \in W$ will be an oscillating one. 
In order to prove Theorem 1 we need the following

LEMMA 1. Let $t_{0} \in \mathbb{R}^{I}$ be an arbitrary fixed point, $\lambda>0$ be an arbitrary constant and $\tau>0$ be a constant delay. We shall assume that the conditions (B) are satisfied:

B1. the function $\varphi(t):\left[t_{0},+\infty\right) \rightarrow[0,+\infty)$ is continuous in the interval $\left[t_{0},+\infty\right)$;

B2. for every $t \geq t_{0}$ the function $\varphi(t)+\lambda \varphi(t-\tau)$ is monotone increasing and $\varphi(t)+\lambda_{\varphi}(t-\tau) \geq C, C>0$.

Then for every $t_{1} \in\left[t_{0},+\infty\right)$ there exists a set

$$
A=\left\{t \mid t_{1} \leq t \leq t_{1}+2 \tau, \lambda \varphi(t-\tau) \geq \beta\right\}
$$

whose measure is not smaller than $\tau$. Here $\beta=\min (C / 2, \lambda C / 2)$.

Proof of Lemma 1. Let $t_{1} \in\left[t_{0},+\infty\right]$ be an arbitrary fixed point and let us consider the set $P=\left\{t \mid t \in\left[t_{1}, t_{1}+\tau\right], \varphi(t)>c / 2\right\}$. If we assume that $P=\emptyset$, then from B2 it follows that the inequality $\lambda \varphi(t-\tau) \geq C / 2$ will hold for every $t \in\left[t_{I}, t_{I}+\tau\right]$ and therefore we could set $A=\left[t_{1}, t_{1}+\tau\right]$.

Let $P \neq \varnothing$ and let us denote by $\alpha, 0<\alpha \leq \tau$, its measure. If we denote by $\bar{P}$ the closure of $P$, then from Bl it follows that the inequality $\varphi(t) \geq C / 2$ will be satisfied for every $t \in \bar{P}$. Let us consider the set $\bar{P}+\tau=\{t \mid t-\tau \in \bar{P}\}$. From B2 it follows that the inequality $\lambda \varphi(t-\tau) \geq \lambda C / 2$ will hold for every $t \in \bar{P}+\tau$. Let us set $A=\left(\left[t_{1}, t_{1}+\tau\right] \backslash P\right) \cup(\bar{P}+\tau)$. Since the measure of $\left[t_{1}, t_{1}+\tau\right] \backslash P$ is $\tau-\alpha$, and the measure of the set $\bar{P}+\tau$ is equal to that of $P$, then the set $A$ will have a measure $\tau$. From the definition of the sets $P$ and $\bar{P}+\tau$ it follows that the inequality $\lambda \varphi(t-\tau) \geq \beta$ holds for every $t \in A$. Thus Lemma 1 has been proved.

Proof of Theorem 1. Let us assume that there exists a non-oscillating solution $x(t)$ of equation (1) belonging to the set $W$. Without loss of generality we consider that there exists a point $\bar{t} \geq t_{0}$ such that 
$x(t)>0$ and $x(t-\tau) \geq 0$ for every $t \geq \bar{t}$. (The case when there exists a point $\bar{t}$ such that $x(\bar{t})<0$ and $x(t-\tau)<0$ for $t \geq \bar{t}$ follows similarly.)

Let us rewrite equation (I) in the form

$$
\left[x^{\prime}(t)+\lambda x^{\prime}(t-\tau)\right]^{\prime}=-F\left(t, x(t-\tau), x^{\prime}(t-\tau)\right) \text {. }
$$

From (3) it follows that the function $x^{\prime}(t)+\lambda x^{\prime}(t-\tau)$ is a monotone decreasing one for $t \geq \bar{t}$. If we assume that there exists a point $t_{2} \geq \bar{t}$ such that

$$
x^{\prime}\left(t_{2}\right)+\lambda x^{\prime}\left(t_{2}-\tau\right)=-C_{1}<0
$$

then for every point $t \geq t_{2}$ the inequality

$$
x^{\prime}(t)+\lambda x^{\prime}(t-\tau) \leq-C_{1}
$$

is satisfied.

Integrating the latter inequality from $t_{2}$ to $t>t_{2}$, we obtain

$$
x(t)+\lambda x(t-\tau) \leq x\left(t_{2}\right)+\lambda x\left(t_{2}-\tau\right)-c_{1}\left(t-t_{2}\right)
$$

After a limit transition $t \rightarrow+\infty$ in inequality (4) we come to a contradiction with the assumption that the function $x(t)$ is non-negative for $t \geq \bar{t}$. Therefore for every point $t \geq \bar{t}$ the following inequality

$$
x^{\prime}(t)+\lambda x^{\prime}(t-\tau) \geq 0
$$

holds. From (3) and (5) it follows that

$$
\begin{aligned}
\lim _{t \rightarrow+\infty} \int_{\bar{t}}^{t} F(s, x(s-\tau), & \left.x^{\prime}(x-\tau)\right) d s \\
= & {\left[x^{\prime}(\bar{t})+\lambda x^{\prime}(\bar{t}-\tau)\right]^{\prime}-\lim _{t \rightarrow+\infty}\left[x^{\prime}(t)+\lambda x^{\prime}(t-\tau)\right]<+\infty . }
\end{aligned}
$$

Furthermore from (5) it follows that $x(t)+\lambda x(t-\tau) \geq c_{2}>0$ for $t \geq \bar{t}$. Then according to Lemma 1 the intersection of the set $E=\left\{t \mid \bar{t} \leq t<+\infty, \lambda x(t-\tau) \geq \beta_{1}\right\}, \beta_{1}=\min \left(C_{2} / 2, \lambda C_{2} / 2\right)$ with the segment $[s, s+2 \tau], s \in[\bar{t},+\infty)$, will have a measure not smaller than $\tau$ and therefore from conditions (A) we shall have 


$$
\int_{E} F\left(t, x(t-\tau), x^{\prime}(t-\tau)\right) d t \geq \inf _{u \geq \beta, \lambda^{-1}} f(u) \int_{E} p(t) d t=+\infty,
$$

which contradicts inequality (6). Thus Theorem 1 has been proved.

DEFINITION 3. The solution $x(t) \in W$ will be called $k_{x}$-oscillating [1] if there exists a number $k_{x} \in \mathbb{R}^{l}$ such that the function $x(t)-k_{x}$ changes its sign in every interval $[\bar{t},+\infty), \bar{t} \geq t_{0}$.

THEOREM 2. Let the conditions AI and A2 of Theorem 1 be satisfied and let the function $p(t):\left[t_{0},+\infty\right) \rightarrow[0,+\infty)$ satisfy

$$
\int_{t_{0}}^{+\infty} p(t) d t=+\infty
$$

Then every solution $x(t) \in W$ wizl be $k_{x}$-oscillating.

In order to prove Theorem 2 we need the following

LEMMA 2. Let the conditions of Theorem 2 be satisfied. Then all non-oscillating solutions of equation (1) belonging to $W$ will have the property $\lim _{t \rightarrow+\infty} \inf |x(t)|=0$.

Proof of Lemma 2. Let us assume that there exists a point. $\bar{t} \geq t_{0}$ such that $x(t) \geq 0$ for $t \geq \bar{t}$. The case when $x(t) \leq 0$ for $t \geq \bar{t}$ is similar. In the proof of Theorem 1 it has been established that if there exists a point $\bar{t} \geq t_{0}$ such that $x(t) \geq 0$ for $t \geq \bar{t}$, the following inequality will hold:

$$
\int_{\bar{t}}^{+\infty} F\left(t, x(t-\tau), x^{\prime}(t-\tau)\right) d t<+\infty .
$$

If we assume that $\lim _{t \rightarrow+\infty} \inf x(t) \geq C_{3}>0$ then there exists a point $t_{4} \geq \bar{t}$ such that $x(t-\tau) \geq c_{3} / 2$ for $t \geq t_{4}$. From (8) it follows that

$$
\inf _{u \geq C_{3} / 2} f(u) \int_{t_{4}}^{+\infty} p(t) d t \leq \int_{t_{4}}^{+\infty} F\left(t, x(t-\tau), x^{\prime}(t-\tau)\right) d t<+\infty
$$


which contradicts equality (7). Thus Lemma 2 has been proved.

Proof of Theorem 2. In the proof of Theorem 1 it has been established that if there exists a point $\bar{t} \geq t_{0}$ such that for $t \geq \bar{t}, x(\bar{t})>0$ and $x(t-\tau) \geq 0$, then

$$
x^{\prime}(t)+\lambda x^{\prime}(t-\tau) \geq 0
$$

holds for every $t \geq \bar{t}$.

From (9) it follows that the function $x(t)+\lambda x(t-\tau)$ is a monotone increasing one and that is why two cases are possible:

(a) $\lim _{t \rightarrow+\infty}[x(t)+\lambda x(t-\tau)]=+\infty$;

(b) $\lim _{t \rightarrow+\infty}[x(t)+\lambda x(t-\tau)]=C_{4}<+\infty$;

(the constant $c_{4}$ can not be zero because $x(t) \in W$ ).

First let us consider (a). Then from Lemma 2 it follows that for every number $k \in(0,+\infty)$ there exists a point $t_{k} \geq \bar{t}$ such that the function $x(t)-k$ changes its sign in every interval $[s,+\infty), s \geq t_{k}$, and therefore $x(t)$ is $k$-oscillating for every $k \in(0,+\infty)$.

For (b) we set

$$
k=C_{3} / 2=\frac{2}{2} \lim _{t \rightarrow+\infty}[x(t)+\lambda x(t-\tau)],
$$

and from Lemma 2 we obtain that $x(t)$ is k-oscillating for $k=c_{3} / 2$.

Thus Theorem 2 has been proved.

Finally we give two examples.

EXAMPLE 1. We consider the equation

$$
x^{\prime \prime}(t)+x^{\prime \prime}(t-2 \pi)+2 x(t-2 \pi)=0 .
$$

In this case $F(t, u, v)=2 f(u), f(u)=u$, and $p(t)=2$. One can immediately verify that the functions $E(t, u, v), f(u)$ and $p(t)$ satisfy the conditions Al-A3 of Theorem 1 and, therefore, all solutions of (10) will be oscillating. (For example, the functions $x(t)=C_{1} \cos t+C_{2} \sin t$ where $C_{1}$ and $C_{2}$ are arbitrary constants, 
$\left|c_{1}\right|+\left|c_{2}\right|>0$, define oscillating solutions of (10).

EXAMPLE 2. In the equation

$$
x^{\prime \prime}(t)+x^{\prime \prime}(t-\tau)+p(t) x^{3}(t-\tau)=0
$$

we have $F(t, u, v)=p(t) f(u), f(u)=u^{3}$, and

$$
p(t)=\left\{\begin{array}{c}
e^{-t}, \quad t \in\left[t_{0}+2 k \tau, t_{0}+(2 k+1) \tau\right], \\
\left(2\left(\tau-e^{-\left[t_{0}+(2 k+2) \tau\right]}\right) / \tau\right)\left[t-\left(t_{0}+(2 k+1) \tau\right)\right]+e^{-\left[t_{0}+(2 k+1) \tau\right]}, \\
t \in\left[t_{0}+(2 k+1) \tau, t_{0}+(2 k+(3 / 2)) \tau\right], \\
\left(2\left(e^{-\left[t_{0}+(2 k+2) \tau\right]}-\tau\right) / \tau\right)\left[t-\left[t_{0}+(2 k+(3 / 2)) \tau\right)\right]+\tau, \\
t \in\left[t_{0}+(2 k+(3 / 2)) \tau, t_{0}+(2 k+2) \tau\right],
\end{array}\right.
$$

$k=0,1,2, \ldots$.

The functions $F(t, u, v)$ and $f(u)$ satisfy the conditions Al and A2 of Theorem 1 , and the function $p(t)\left(p(t)>0\right.$ for $\left.t \geq t_{0}\right)$ satisfies $(7)$. Then, from Theorem 2, it follows that there exists numbers $k$, $k \in(-\infty,+\infty)$ such that all the solutions of (1l) are $k$-oscillating.

Note. The function $p(t)$ from (11) is an example of a function satisfying the conditions of Theorem 2 but not satisfying the condition $A 3$ of Theorem 1 .

Actually, let us denote by $E$ the set

$$
E=\bigcup_{k=0}^{\infty}\left[t_{0}+2 k \tau, t_{0}+(2 k+1) \tau\right]
$$

whose intersection with every interval of the form $[t, t+2 \tau]$ has a measure $\tau$; then

$$
\int_{E} p(t) d t=\int_{E} e^{-t} d t \leq \int_{t_{0}}^{+\infty} e^{-t}<+\infty .
$$




\section{References}

[1] С.Б. Норнин [S.B. Norkin], "Осцилляция речений дифференциальных уравненнй с отнлонянщмся аргументом" [Oscillation of the solutions of differential equations with a deviating argument], дияберенииальние уравнения с отклонялимся аргументом, 247-256 [Differential equations with a deviating argument] (Naukova Dumka, Kiev, 1977).

[2] В.Н. Шевело [V.N. Ševelo], осиилляиия решений оифференииальных

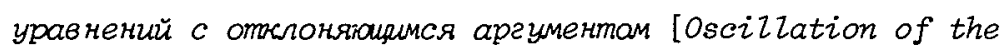
solutions of differential equations with a deviating argument] (Naukova Dumka, Kiev, 1978).

\footnotetext{
Faculty of Mathematics,

University of Plovdiv,

Car Asen Str. 24,

4000 Plovdiv,

Bulgaria.
} 